Number of pages: 20

Number of Figures: 10

Number of Tables: 2

Number of References: 21

\title{
FREQUENCY-DEPENDENCE OF DISCOMFORT CAUSED BY VIBRATION AND MECHANICAL SHOCKS
}

\author{
Giulia Patelli, Miyuki Morioka and Michael J. Griffin \\ Human Factors Research Unit \\ Institute of Sound and Vibration Research \\ University of Southampton, \\ Southampton SO17 1BJ
}

England

Address for correspondence:

Professor Michael J Griffin

e-mail: M.J.Griffin@soton.ac.uk

Telephone: +44 (0)23 80592277

Facsimile: +44 (0)23 80592927 


\section{Abstract}

The frequency content of a mechanical shock is not confined to its fundamental frequency, so it was hypothesized that the frequency-dependence of discomfort caused by shocks with defined fundamental frequencies will differ from the frequency-dependence of sinusoidal vibration. Subjects experienced vertical vibration and vertical shocks with fundamental frequencies from 0.5 to $16 \mathrm{~Hz}$ and magnitudes from \pm 0.7 to $\pm 9.5 \mathrm{~ms}^{-2}$. The rate of growth of discomfort with increasing magnitude of motion decreased with increasing frequency of both motions, so the frequency-dependence of discomfort varied with the magnitudes of both motions and no single frequency weighting will be ideal for all magnitudes. At the frequencies of sinusoidal vibration producing greatest discomfort (4 to $16 \mathrm{~Hz}$ ), shocks produced less discomfort than vibration with same peak acceleration or unweighted vibration dose value. Frequency-weighted vibration dose values provided the best predictions of the discomfort caused by different frequencies and magnitudes of vibration and shock.

\section{Practitioner Summary}

Human responses to vibration and shock vary according to the frequency content of the motion. The ideal frequency weighting depends on the magnitude of the motion. Standardised frequency-weighted vibration dose values estimate discomfort caused by vibration and shock but for motions containing very low frequencies the filtering is not optimum.

Keywords: Vibration comfort; whole-body vibration; mechanical shocks; frequency weightings; vibration standards 


\section{Introduction}

The discomfort caused by whole-body oscillatory motion depends on the frequency content of the motion and so 'frequency weightings' are used to give more weight to those frequencies causing greater discomfort. Standards give guidance on the use of frequency weightings for evaluating oscillatory motions with respect to discomfort and risk of injury. The frequency weightings in current standards (British Standard 6841:1987 and International Standard 2631-1:1997) have been greatly influenced by studies of vibration discomfort caused by sinusoidal vibration (e.g., Griffin et al., 1982; Corbridge and Griffin 1986), but people are mostly exposed to non-sinusoidal motions.

Many environments expose people to mechanical shocks that vary in their magnitude and their frequency content: the two characteristics that influence the waveform of a motion. Whereas sinusoidal vibration is dominated by a single frequency, a mechanical shock always has a frequency content that includes a wide range of frequencies. The discomfort caused by vertical sinusoidal vibration has been reported by Zhou and Griffin (2014b) and the discomfort caused by vertical mechanical shocks has been reported by Zhou and Griffin (2017b). Over the frequency range 1 to $16 \mathrm{~Hz}$ their studies confirmed that with increasing magnitude of both types of motion the rate of growth of discomfort decreased as the fundamental frequency of the motion increased, consistent with some previous studies (e.g. Morioka and Griffin, 2006a,b; Ahn and Griffin, 2008). The frequencydependence in the rate of growth causes the frequency-dependence of vibration discomfort (as shown in equivalent comfort contours) to change with the magnitude of the motion. The two studies by Zhou and Griffin with vibration and shock used different subjects and settings, so it was not meaningful to test for any differences in either the magnitude-dependence or the frequency-dependence of discomfort caused by shocks and vibration, or compare the body locations experiencing greatest discomfort. Zhou and Griffin (2014b, 2017b) only investigated the frequency-dependence of discomfort for frequencies greater than $1 \mathrm{~Hz}$, even though their shocks contained frequencies less than $1 \mathrm{~Hz}$.

A shock contains many frequency components, with the shock waveform determined by the magnitude of each frequency component and the phase between the frequency components. There will be components at frequencies greater than the nominal frequency of a shock and components at frequencies less than the nominal frequency of the shock. This means that the discomfort caused by a shock will depend on sensitivity to vibration at frequencies both greater than and less than the fundamental frequency of the shock, not only on sensitivity to vibration at the nominal frequency of the shock. A shock with a specific nominal frequency would be expected to cause less discomfort than expected for its magnitude if there is high sensitivity to that frequency, because some of the shock 
magnitude is at frequencies where there is less sensitivity. Similarly, a shock might cause greater discomfort than expected for its magnitude if the frequency of greatest sensitivity is at another nearby frequency. These effects would be predicted by a suitable frequency weighting, but there are no known experimental studies to test the expectation.

The relevant frequency weightings in the current standards are intended for motions in the frequency range 0.5 to $80 \mathrm{~Hz}$ and have band-pass filters (high-pass at $0.4 \mathrm{~Hz}$ and lowpass $100 \mathrm{~Hz}$ ) that attenuate motion outside this frequency range. The high-pass and lowpass filters will attenuate components in the waveform of shocks with energy close to, or outside, the 0.4 to $100 \mathrm{~Hz}$ frequency band. This will reduce the calculated vibration dose values for such shocks and so underestimate their severity. The phase between frequency components in a vibration can also influence judgements of discomfort (Matsumoto and Griffin, 2002), but the phases of the standardised frequency weighting filters and bandpass filters arise solely from convenience and not evidence of the phase response appropriate for predicting vibration discomfort. The phase characteristics of filters do not affect r.m.s. values but they do affect the peak values and vibration dose values that are recommended for evaluating shocks and other transients (BS 6841:1987; ISO 2631$1: 1997)$.

This paper is based on an experiment conducted in partial fulfilment of a PhD thesis (Patelli, 2016). The experiment was designed to investigate whether, for motions having fundamental frequencies in the range 0.5 to $16 \mathrm{~Hz}$, the magnitude-dependence and the frequency-dependence of the discomfort caused by $1 \frac{1}{2}$-cycle shocks differs from the magnitude-dependence and the frequency-dependence of the discomfort caused by sinusoidal vibration. To understand possible causes of any differences between the discomfort caused by sinusoidal vibration and mechanical shocks, differences in the body locations of greatest discomfort were also investigated. The effects of the frequency weighting filters and the band-pass filters on the evaluation of mechanical shocks having fundamental frequencies less than $1.0 \mathrm{~Hz}$ were also investigated.

It was hypothesized that for both sinusoidal vibration and mechanical shocks the rate of growth of discomfort would decrease as the fundamental frequency increased from 0.5 to $16 \mathrm{~Hz}$. It was hypothesized that at frequencies causing greatest discomfort, sinusoidal vibration would cause greater discomfort than shocks having the same fundamental frequency and the same unweighted vibration dose value, VDV. 


\section{Method}

\section{$2.1 \quad$ Subjects}

Eighteen male students and office workers at the University of Southampton participated in the study. They were aged 20 to 37 years, had statures between 166 and $189 \mathrm{~cm}$, and weights between 59 and $91 \mathrm{~kg}$. Results are presented for only 17 subjects because one subject withdrew from the study for personal reasons. The experiment was approved by the Ethics Committee of the Faculty of Engineering and the Environment at the University of Southampton (Reference number: 8630).

\subsection{Apparatus}

Vertical oscillations were produced by a 1-m stroke vertical electrohydraulic vibrator (Servotest Testing Systems Ltd., Surrey, UK). Mechanical shocks and sinusoidal vibrations were generated by the HVLab Matlab Toolbox (version 2.0, ISVR, University of Southampton, UK), and then equalized and reproduced by a Servotest Pulsar system.

A rigid flat seat was mounted on the platform of the vibrator. The seat had an upright rigid backrest but subjects were asked not to make contact with the backrest. The height, width, and depth of the horizontal supporting seat surface were $41 \mathrm{~cm}$ by $71 \mathrm{~cm}$ by $51 \mathrm{~cm}$, respectively. There was no backrest contact and the influence of vibration at the feet was ignored. A noise system (HFRU Noise system 001, ISVR, University of Southampton, UK) produced white noise at approximately $75 \mathrm{dBA}$ via a pair of headphones so as to mask any small variations in the noise of the vibrator.

\section{$2.3 \quad$ Stimuli}

The motions were vertical sinusoidal vibrations and vertical mechanical shocks with fundamental frequencies at the 16 preferred one-third octave centre frequencies in the range 0.5 to $16 \mathrm{~Hz}$. All motions were produced at a sampling rate of 512 samples per second and the resulting acceleration sampled at 512 samples per second via $100-\mathrm{Hz}$ low pass filters

All 'sinusoidal' vibration stimuli had durations of $5 \mathrm{~s}$, with the magnitude of the sinusoid multiplied by the first $5 \mathrm{~s}$ of a $0.1-\mathrm{Hz}$ sinusoid. This provided a smooth start and end to the vibrations with maximum magnitude around $2.5 \mathrm{~s}$ (Figure 1).

The shocks were formed from $1 \frac{1}{2}$ cycles of a sinusoid of the required fundamental frequency multiplied by a half cosine over the duration of the $1 \frac{1}{2}$-cycle sinusoid (Figure 1 ). The durations of the shocks therefore depended on their fundamental frequency, 
decreasing from $3 \mathrm{~s}$ at $0.5 \mathrm{~Hz}$ to $0.09 \mathrm{~s}$ at $16 \mathrm{~Hz}$. The frequency components within shocks having this waveform are distributed above and below the fundamental frequency of each shock as shown in Figure 10 of Zhou and Griffin (2017b).

The shocks were presented so that the motion moved up from the starting position and then returned to the starting position (Figure 1). A previous study with shocks having the same waveform and similar magnitudes found no significant difference in the discomfort caused by 'upward' and 'downward' shocks (Zhou and Griffin, 2017b).

\section{FIGURE 1 ABOUT HERE}

The magnitudes of all motions were quantified in terms of vibration dose value, VDV, either unweighted or frequency-weighted using weighting $W_{\mathrm{b}}$ in accord with BS 6841:1987 and ISO 8041:2005. A slightly different frequency weighting, $W_{\mathrm{k}}$, is included in ISO 2631$1: 1997$ but unlike frequency weighting $W_{b}$ it is not traceable to an experimental origin. Irrespective of the fundamental frequency, or whether the motion was vibration or shock, motions with similar frequency-weighted VDVs would be expected to produce broadly similar discomfort.

For both vibration and shock, at each fundamental frequency, eight magnitudes of frequency-weighted VDV were presented in $2 \mathrm{~dB}$ steps. The magnitudes varied with the frequency of motion but were always within the range 0.16 to $4.0 \mathrm{~ms}^{-1.75}\left(W_{\mathrm{b}}\right.$-weighted VDV). This range of vibration magnitudes falls within the range 'not uncomfortable' to 'very uncomfortable' according to BS 6841:1987 and is typical of magnitudes of vibration and shock found in public transport. Table 1 shows the magnitudes of all the stimuli in terms of unweighted r.m.s. acceleration (for vibration) and unweighted peak acceleration (for shock). The ranges of magnitudes of the stimuli at each frequency are also shown for unweighted VDV in Figure 4, below, and for peak acceleration in Figure 5, below.

\section{TABLE 1 ABOUT HERE}

\section{$2.4 \quad$ Procedure}

Subjects attended two sessions. Half of the subjects commenced with a session in which they experienced vibration, and the others commenced with a session in which they experienced shocks.

Subjects sat in comfortable upright postures without touching the backrest and wearing a loose lap belt (Figure 2). Their hands rested on their laps and their eyes were closed. The angle between the thighs and the calves was about 90 degrees with the feet parallel and 
resting on the platform of the vibrator. Subjects wore the headphones presenting approximately $75 \mathrm{dBA}$ of white noise during the whole duration of the experiment.

\section{FIGURE 2 ABOUT HERE}

Prior to commencing the experiment, subjects were provided training on how to report their judgements of discomfort. The method of magnitude estimation was explained and then subjects practiced by giving numerical ratings of the apparent lengths of lines of different length. Subjects could give any number for the apparent length of the first line (although 100 was suggested), and then rated the apparent length of other lines so that a line appearing to be twice the length of the first line was assigned double the number assigned to the first line (i.e., 200), and so on.

Subjects also rated their discomfort caused by each motion using absolute magnitude estimation. In both sessions, the first motion they experienced was a vibration close to the 'middle' frequency and the 'middle' magnitude of the sinusoidal vibrations (i.e., a 5-s motion with a frequency of $2.5 \mathrm{~Hz}$, an unweighted magnitude of $1.1 \mathrm{~ms}^{-2}$ r.m.s., unweighted VDV of $2.0 \mathrm{~ms}^{-1.75}$, and $W_{\mathrm{b}}$-weighted VDV of $\left.1.0 \mathrm{~ms}^{-1.75}\right)$. They judged the discomfort caused by all other stimuli (both vibrations and shocks) in proportion to the number they provided for the discomfort caused by this first sinusoidal motion. If they were unsure of their rating of discomfort, the motion was repeated.

After every stimulus, the subjects also indicated the part of the body where they felt greatest discomfort by referring to the body map shown in Figure 2.

Subjects were first exposed to a sequence of 14 practice stimuli, covering the range of magnitudes and frequencies they would judge in the session. In each session, they were then exposed to 128 motion stimuli.

\section{$2.5 \quad$ Statistical analysis}

Non-parametric tests were used in the statistical analysis. To investigate differences between related samples, the Friedman two-way analysis of variance and the Wilcoxon matched-pairs signed ranks were used. The significance levels reported have not been adjusted for multiple comparisons. To investigate whether the locations of greatest discomfort changed with the frequency or waveform of vibration, the Cochran $Q$ test and the McNemar test were used. These tests were applied to binary data, with a body location assigned ' 1 ' for an occurrence and ' 0 ' otherwise. 


\section{Results}

\subsection{Rate of growth of discomfort}

For a stimulus having a physical magnitude, $\varphi$, Stevens' power law assumes the sensation, $\psi$, is proportional to the physical magnitude raised to a power, $n$ :

$$
\psi=k \cdot \varphi^{n}
$$

In this study, $\psi$ was the magnitude estimate of discomfort and $\varphi$ was the vibration dose value of the stimulus.

The rate of growth of discomfort, $n$, was determined for every subject and all fundamental frequencies of both the sinusoidal vibrations and the shocks by linear regression after logarithmic transformation of equation (1):

$$
\log _{10} \psi=n \log _{10} \varphi+\log _{10} k
$$

For vibration and shock, the rate of growth of discomfort, $n$, depended on the fundamental frequency of the motion (Table 2; Figure 3; $p<0.001$, Friedman), with a trend for decreased rate of growth with increased frequency.

\section{TABLE 2 ABOUT HERE}

\section{FIGURE 3 ABOUT HERE}

The rate of growth of discomfort was greater for shock than for vibration at $0.63,0.8,2.0$, $3.15,5.0,6.3$, and $12.5 \mathrm{~Hz}$ ( $p<0.012$; Wilcoxon) and at $16 \mathrm{~Hz}$ ( $p=0.044$; Wilcoxon).

Over all subjects and all stimuli, the median value of the Spearman rank correlation coefficient, $r_{\mathrm{s}}$, was 0.86 for the vibration stimuli and 0.89 for the shock stimuli, with interquartile ranges of 0.78 to 0.92 and 082 to 0.93 , respectively.

\subsection{Equivalent Comfort Contours}

\subsubsection{Equivalent comfort contours in terms of unweighted VDV}

From the rate of growth of discomfort, $n$, and the constant, $k$, the unweighted vibration dose value required by each subject at each frequency to produce subjective magnitudes, $\psi$, from 63 to 160, were calculated using Equation 2 (where a subjective magnitude of 100 corresponds to the discomfort experienced with a 5-s motion with a frequency of $2.5 \mathrm{~Hz}$ and $W_{\mathrm{b}}$-weighted VDV of $\left.1.0 \mathrm{~ms}^{-1.75}\right)$. 
For both shock and vibration, the shapes of the median equivalent comfort contours changed as the subjective magnitude increased from 63 to 160 (Figure 4). This change is a consequence of the frequency-dependence in the rate of growth of discomfort shown in Figure 3.

\section{FIGURE 4 ABOUT HERE}

With both waveforms, the unweighted vibration dose value required for a subjective magnitude of 100 (i.e., $\psi=100$ ) was strongly dependent on the frequency of the vibration $(p<0.001$, Friedman). With vibration, sensitivity at $5 \mathrm{~Hz}$ was significantly greater than all other frequencies in the range 0.5 to $8 \mathrm{~Hz}(p<0.031$, Wilcoxon).

When expressed in terms of the unweighted VDV, the equivalent comfort contours for shocks exhibit a flatter shape than the contours for vibration (Figure 4). The unweighted VDV required for a subjective magnitude of 100 was greater for shocks than for vibration at all frequencies from 4.0 to $16 \mathrm{~Hz}$ (Wilcoxon, $p<0.013$ ), except at $12.5 \mathrm{~Hz}$ (Wilcoxon, $p=0.062)$.

\subsubsection{Equivalent comfort contours in terms of unweighted peak acceleration}

When expressed in terms of the unweighted peak acceleration, the contours show trends similar to those with unweighted VDV, but with greater differences between the vibration and the shock at frequencies greater than $5 \mathrm{~Hz}$ (Figure 5).

\section{FIGURE 5 ABOUT HERE}

\subsubsection{Equivalent comfort contours in terms of frequency-weighted VDV}

With both waveforms, the weighted vibration dose value required for a subjective magnitude of 100 (i.e., $\psi=100$ ) was also dependent on the frequency of the vibration $(p<0.001$, Friedman).

For a subjective magnitude of 100 , the frequency-weighted VDV required for similar discomfort was greater for shocks than for vibration at all frequencies from 4 to $16 \mathrm{~Hz}$ $(p<0.016$; Wilcoxon, Figure 6), except at $12.5 \mathrm{~Hz}$ ( $p=0.121$; Wilcoxon). The frequencyweighted VDV required for a subjective magnitude of 100 was slightly less for shocks than for vibration at frequencies from $0.5 \mathrm{~Hz}$ to $0.8 \mathrm{~Hz}(p<0.036)$.

\section{FIGURE 6 ABOUT HERE}




\subsection{Locations of discomfort}

With both waveforms, as the frequency increased from 0.5 to $16 \mathrm{~Hz}$, the location of greatest discomfort tended to fall from the upper body (head and torso) to the lower body (buttocks, thighs, calves and feet). This is shown for the lowest, middle, and highest magnitudes in Figure 7 where the ordinate is a percentage, with $100 \%$ corresponding to all 17 subjects. The height of each bar shows the percentage of subjects choosing the location for that bar. At the middle and highest magnitudes of vibration, the location of greatest discomfort varied with the frequency of vibration (location $A, p<0.001$; location $B$, $p<0.032$; location $C, p<0.001)$. With shocks, the location of greatest discomfort also varied with the fundamental frequency of the shock (middle magnitude: location $A, p<0.001$; location $C, p<0.001$; highest magnitude: location $A, p<0.001$; location $B, p=0.008$; and location $\mathrm{C}, p<0.001$; Cochran).

\section{FIGURE 7 ABOUT HERE}

At each frequency, the location of greatest discomfort was not significantly different for vibration and shock, except with the middle magnitude at $5 \mathrm{~Hz}$ (where location $\mathrm{C}$ buttocks and legs - was more frequent with shock than with vibration, $p=0.016$; McNemar), and at both $5 \mathrm{~Hz}$ and $6.3 \mathrm{~Hz}$ (where location $\mathrm{B}$ - thorax - was more frequent with vibration than with shocks, $p<0.016$ ). With the highest magnitude at $3.15 \mathrm{~Hz}$, location $C$ (buttocks and legs) was more frequently the location of greatest discomfort during shock than during vibration $(p=0.004)$.

\section{Discussion}

\subsection{Rate of growth of discomfort}

The value of $k$ (in Equation 1) depends on the units of vibration magnitude employed (e.g., peak acceleration, r.m.s. acceleration, or vibration dose value) and shows the frequencydependence of vibration discomfort when the vibration magnitude, $\varphi$, is unity (e.g., when the peak acceleration is $1.0 \mathrm{~ms}^{-2}$ or when the vibration dose value is $\left.1.0 \mathrm{~ms}^{-1.75}\right)$. The reciprocal of $k$ is therefore the frequency weighting for vibration discomfort at unity magnitude.

The value of $n$ (in Equation 1) reflects how vibration discomfort changes as the vibration magnitude increases above, or falls below, unity magnitude. If $n$ depends on the frequency of vibration the shapes of the equivalent comfort contours change as the vibration magnitude changes, and the appropriate frequency weightings also change with vibration magnitude. 


\subsubsection{Vibration}

The rate of growth of discomfort caused by vibration depended on the frequency of the vibration (Figure 3). As found previously, the rate of growth decreased as the frequency of vibration increased from 0.5 to $2 \mathrm{~Hz}$ (e.g., for vertical vibration, Zhou and Griffin, 2014b; for horizontal vibration, Wyllie and Griffin, 2009). For 1 to $16 \mathrm{~Hz}$ sinusoidal vertical vibration over the range 0.1 to $4.0 \mathrm{~ms}^{-2}$ r.m.s., Zhou and Griffin (2014b) found that at any frequency in the range 1 to $5 \mathrm{~Hz}$ the rate of growth was greater than at any frequency in the range 6.3 to $16 \mathrm{~Hz}$.

\subsubsection{Shocks}

The rate of growth of discomfort caused by shock also decreased as the fundamental frequency of the shocks increased (Figure 3). There have been fewer studies with shock than with vibration but both Ahn and Griffin (2008) and Zhou and Griffin (2017b) also found the rate of growth decreased as the fundamental frequencies of shocks increased (Figure 8). Like the present study, Ahn and Griffin (2008) found that the greatest change in the rate of growth for shocks occurred at frequencies less than about $2 \mathrm{~Hz}$. All three studies have found that the rate of growth of discomfort caused by shocks is less dependent on frequency with fundamental frequencies greater than about $4 \mathrm{~Hz}$. The reasons for differences in the rates of growth of discomfort in the study by Ahn and Griffin (2008) are unknown but may be related differences in the ranges of discomfort experienced by subjects, differences in the shock waveforms, and other factors.

\section{FIGURE 8 ABOUT HERE}

For shocks with fundamental frequencies from 1 to $16 \mathrm{~Hz}$ over a range of magnitudes from \pm 0.1 to $\pm 7.9 \mathrm{~ms}^{-2}$ (corresponding to $W_{\mathrm{b}}$-weighted vibration dose values from 0.05 to 2.0 $\mathrm{ms}^{-1.75}$ ), Zhou and Griffin (2017b) found no significant difference in the rate of growth of discomfort between upward and downward shocks, or between low magnitude and high magnitude shocks.

\subsubsection{Comparison of vibration and shock}

The present study was designed to allow a direct comparison of the rate of growth of discomfort for vibration and shock having the same fundamental frequency and the same range of $W_{b}$-weighted VDV. Previously, separate experiments with sinusoidal vibration (Zhou and Griffin, 2014b) and with shock (Zhou and Griffin, 2017b) were conducted with different ranges of magnitudes. Although the studies of Zhou and Griffin (2014b) suggest the rate of growth of discomfort does not greatly depend on the magnitude of vibration or 
shock (over the ranges investigated) it may depend on the range of magnitudes of the stimuli being judged. The studies of Zhou and Griffin employed different subjects (20 males and 20 females with vibration but only 20 males with shock) and were not designed to compare subjective responses to sinusoidal vibration and shock.

The rates of growth of discomfort found in the present study were generally greater for shock than for vibration (Figure 3 , Table 2). A shock with a fundamental frequency $f_{0}$ contained components at frequencies less than, and greater than, $f_{0}$ (see Figure 10 in Zhou and Griffin 2017b), so its rate of growth is expected to depend on the rate of growth over a range of frequencies lower and higher than the fundamental frequency of the shock. The frequency distribution of energy in a shock is dependent on the shock waveform, so it is not possible to conclude that the rate of growth of discomfort for shocks is always greater than, or less than, the rate of growth of discomfort for vibration. Although there were greater values of $n$ for shocks than for vibration in this study, the difference may not be of prime importance because the rate of growth of discomfort is more dependent on the frequency content of the motion than whether it is vibration or shock.

\subsection{Equivalent comfort contours}

\subsubsection{In terms of unweighted VDV}

The reduction in the rate of growth of discomfort, $n$, with increasing frequency of vibration means that the frequency-dependence of discomfort caused by vibration depends on the magnitude of motion, with equivalent comfort contours being more widely separated at frequencies greater than $2 \mathrm{~Hz}$ than at frequencies less than $2 \mathrm{~Hz}$ (Figure 4).

For a subjective magnitude of 100 , greatest sensitivity (i.e., where the acceleration required to cause a given level of discomfort is least) was at $5 \mathrm{~Hz}$, consistent with previous studies using a variety of psychophysical methods for evaluating vibration discomfort (e.g., Dupuis et al., 1972; Jones and Saunders, 1972; Griffin, 1976; Griffin et al., 1982; Corbridge and Griffin, 1986; Morioka and Griffin, 2006a; Zhou and Griffin, 2014b). These studies investigated various ranges of vibration magnitude, vibration frequency, and durations of vibration, but the contours have broadly similar shapes, with the exception of the reduced sensitivity to higher frequencies in the studies reported by Dupuis et al. (1972) and Jones and Saunders (1972) (see Figure 10 in Zhou and Griffin, 2014b).

The frequency-dependence in the rate of growth of discomfort also caused the equivalent comfort contours for shock to change shape according to the magnitude of the shock (Figure 4). 


\subsubsection{Comparing equivalent comfort contours for vibration and shock in terms of unweighted acceleration}

The VDV, r.m.s., and peak of a motion are fundamentally different measures that give different indications of the severity of a shock. The peak value of a shock is the same irrespective of how many times a person is exposed to the peak value, and it is unaffected by the duration of a shock or the acceleration at magnitudes less than the peak value. This is not representative of how shocks or vibration cause discomfort. The equivalent comfort contours for shocks showed similar trends for VDV and peak, but with differences because the VDV depends on the duration of the motion and it is influenced by magnitudes less than the peak value.

The measurement duration affects an r.m.s. measure of a shock because, being an average, the r.m.s. reduces as the duration increases beyond the duration of the event. The r.m.s. acceleration is not appropriate for evaluating shocks because the duration of measurement cannot be defined in a practically useful way. The measurement duration is less important with statistically stationary motions because a sufficient period can be considered representative of any other period, including the complete motion. However, with a statistically stationary motion a short duration will have the same r.m.s. value as a long duration, even though a long duration will cause more discomfort. Because it is an accumulative measure and not an average measure, the VDV does not depend on the measurement duration if the measurement period includes all of the motion of interest. The vibration dose value of a long period of statistically stationary motion has a greater value than a short period, with the increase assumed to represent the increase in discomfort.

The inherent differences between the VDV, the r.m.s., and peak value, affect the shape of an equivalent comfort contour for shock, but not the shape of an equivalent comfort contour for a fixed duration of vibration. When using unweighted peak acceleration (Figure 5 ), there are greater differences between the contours for shock and the contours for vibration than when using the unweighted VDV (Figure 4). This suggests the VDV is a better indicator of discomfort than peak acceleration when no frequency weighting is employed.

For fundamental frequencies greater than about $4 \mathrm{~Hz}$, there was greater sensitivity to the unweighted VDV of the sinusoidal vibrations than to the unweighted VDV of the shocks (Figure 4).

Whereas all the sinusoidal motions had durations of 5 seconds, the duration of each shock was $n_{\mathrm{c}} / f_{0}$, where $f_{0}$ is the fundamental frequency of the shock and $n_{\mathrm{c}}$ is the number of 
cycles $\left(1 \frac{1}{2}\right.$ cycles for the shocks employed in this study). The durations of the shocks therefore reduced from $3 \mathrm{~s}$ (for $0.5-\mathrm{Hz}$ shocks) to $0.09 \mathrm{~s}$ (for $16-\mathrm{Hz}$ shocks). The VDV assumes discomfort depends on the $4^{\text {th }}$ root of the duration of motion, and that discomfort is similar if the VDV is similar. The 32-fold reduction in shock duration (from 0.5 to $16 \mathrm{~Hz}$ ) will have resulted in a 2.38 -fold reduction in the VDV for $16-\mathrm{Hz}$ shocks compared to $0.5-$ $\mathrm{Hz}$ shocks. The effect of the time-dependency continues progressively from 0.5 to $16 \mathrm{~Hz}$, whereas the difference between the equivalent comfort contours for vibration and shock is not progressive from 0.5 to $16 \mathrm{~Hz}$. It follows that differences between the equivalent comfort contours for vibration and shock in Figure 4 cannot be explained solely by the effect of duration on discomfort being inadequately reflected in the fourth-power vibration dose value (unless it is assumed that a different time-dependence is needed at different frequencies).

The different frequency-dependence evident in the contours for unweighted vibration and unweighted shock will be due, at least in part, to the shocks containing energy at frequencies other than their fundamental frequency. For shocks having their fundamental frequency where there is greatest sensitivity to vibration (5 to $16 \mathrm{~Hz}$ in this study, depending on the magnitude of the motion; Figure 4), there will be energy in the shocks at lower and higher frequencies, where sensitivity is less. So, when the fundamental frequency of a shock is at a frequency of greatest sensitivity, a greater unweighted VDV will be required to produce the same discomfort as caused by a sinusoidal motion of that frequency. Although the frequency-dependence of discomfort caused by shocks will depend on the shock waveform, the results are consistent with the hypothesis that when the fundamental frequency of a shock is at a frequency where sensitivity to vibration is high, the shock will produce less discomfort than a sinusoidal vibration with the same frequency and the same magnitude (e.g., the same unweighted vibration dose value).

The equivalent comfort contours for shock and vibration determined in this study can be compared with equivalent comfort contours obtained by Zhou and Griffin (2014b, 2017b) in their 'high magnitude sessions' (Figure 9). Although the contours are not identical, they show similar characteristics. In this study the subjects sat in comfortable upright postures without touching a backrest and they wore a loose lap belt. Sitting posture and contact with a backrest can alter the frequency-dependence of discomfort caused by vertical vibration (Basri and Griffin, 2013) and it might be expected that with greater magnitude shocks the tightness of a belt may alter discomfort. These factors need to be taken into account when applying the findings of the study 


\subsubsection{Comparing equivalent comfort contours for vibration and shock in terms of frequency-weighted vibration dose values}

When the equivalent comfort contours for vibration and shock are expressed in terms of frequency-weighted VDV, using weighting $W_{\mathrm{b}}$ as shown in Figure 6 , they should be horizontal if the frequency weighting and the time-dependence in the evaluation method are both appropriate.

The equivalent comfort contours in terms of frequency-weighted VDV have somewhat flatter shapes than the unweighted contours in Figure 4 but they are not horizontal. At frequencies greater than $1 \mathrm{~Hz}$, the contours for sinusoidal vibration tend to either increase or decrease with increasing frequency, depending on the vibration magnitude. This is caused by the frequency-dependence of the rate of growth of discomfort and so, although it may be argued that the $W_{\mathrm{b}}$ frequency weighting is a reasonable compromise for the magnitudes of vibration used in this study, it may not be optimum for much higher or much lower magnitudes.

The equivalent comfort contours for shock show a progressive rise in frequency-weighted VDV with increasing frequency, suggesting greater sensitivity at the lower frequencies (or less sensitivity at the higher frequencies) than predicted by their frequency-weighted VDV. The rise in sensitivity to frequency-weighted VDV at low frequencies is consistent with the findings of both Ahn and Griffin (2008) and Zhou Griffin (2017b). With shocks having frequency-weighted VDVs around $2.9 \mathrm{~ms}^{-1.75}$, Ahn and Griffin (2008) found greater than expected discomfort at frequencies less than about $2 \mathrm{~Hz}$. For shocks with a frequency weighted VDV of $2.0 \mathrm{~ms}^{-1.75}$, Zhou and Griffin (2017b) found that magnitude estimates of discomfort tended to decrease over the frequency range 1 to $4 \mathrm{~Hz}$ and then remain relatively constant at frequencies greater than $4 \mathrm{~Hz}$. The findings of both studies might appear to suggest that for this magnitude of shock there is a need for greater gain in the frequency weighting than given by frequency weighting $W_{b}$ at frequencies less than around $2 \mathrm{~Hz}$.

The present and previous studies are consistent in indicating that the rate of growth of discomfort for shocks is greater at frequencies less than $2 \mathrm{~Hz}$ and that discomfort caused by low frequency shocks is greater than predicted by frequency weighting $W_{\mathrm{b}}$ (see Section 4.1). In part, the underestimation of discomfort at these low frequencies might be attributed to the relatively high magnitude of the shocks compared to the magnitudes of vibration that influenced the shape of the $W_{\mathrm{b}}$ frequency weighting (equivalent to 0.25 and $0.75 \mathrm{~ms}^{-}$ 2 r.m.s. at $2 \mathrm{~Hz}$; Corbridge and Griffin, 1986). The displacements associated with the low frequency motions were much greater than in previous studies with sinusoidal motion and 
they will have caused a different type of vestibular excitation as well as various somatosensory sensations.

The present study investigated greater magnitudes of sinusoidal vibration than Zhou and Griffin (2014b) but both studies found that increasing the magnitude of the vibration decreased the frequency of greatest sensitivity to vibration. The shapes of the equivalent comfort contours for shock also changed with the magnitude of the shock, but both Zhou and Griffin (2017b) and the present study found flatter equivalent comfort contours for shocks than for vibration, when the motion magnitude is expressed in terms of unweighted vibration dose value (Figure 4).

The 'nonlinearity' in the frequency-dependence of vibration discomfort is partly caused by nonlinearity in biodynamic responses (Matsumoto and Griffin, 2002). The nonlinearities in biodynamic responses to vertical vibration have been shown to vary with the frequency of vertical vibration, although only at frequencies greater than about $2.5 \mathrm{~Hz}$ (Zhou and Griffin, 2014a). The optimum stiffness of a simple biodynamic model representing the vertical apparent mass of the body exposed to vertical shocks of constant vibration dose value, increased for shocks having fundamental frequencies greater than $3.15 \mathrm{~Hz}$, with the optimum stiffness more dependent on the fundamental frequency of a shock than the magnitude of a shock (Zhou and Griffin, 2017a). Considering the large differences in the physical characteristics of the stimuli (as shown in Table 1), these nonlinearities may partly explain why the equivalent contours for vibration and shock in Figure 6 differ over the range 2.5 to $16 \mathrm{~Hz}$.

Many frequency components of different magnitudes combine to form a mechanical shock. Since the magnitude of sinusoidal vibration affects the frequency-dependence of response to single frequency motions, it is not surprising that the magnitude of a shock affects the frequency-dependence of responses to shocks. However, the magnitudes of the many frequency components in a shock are lower than the single frequency component in a sinusoidal motion that has the same magnitude as the shock. It is therefore unclear what equivalent comfort contour is likely to be most appropriate for defining a frequency weighting for any shock, let alone all shocks. The previous and the present studies indicate that the use of a single frequency weighting cannot be expected to provide an accurate prediction of the discomfort caused by all possible shocks. Shocks with different waveforms have different spectra, so it is not appropriate to derive a frequency weighting for the fundamental frequency of one waveform and apply it to shocks having different waveforms. 


\subsection{Locations of discomfort}

Shocks and vibration showed similar locations of greatest discomfort. With frequencies less than about $2.5 \mathrm{~Hz}$, discomfort was mostly felt in the chest and abdomen, whereas with frequencies greater than $6.3 \mathrm{~Hz}$ discomfort was mostly felt in the buttocks, legs, and feet. With frequencies in the range $3.15 \mathrm{~Hz}$ to $6.3 \mathrm{~Hz}$, greatest discomfort was in the upper body for vibration but in the lower body for shocks, but with significant differences between the two waveforms only at $5 \mathrm{~Hz}$. The findings indicate that the broader frequency spectrum of the mechanical shocks used in this study did not greatly change the transmission of motion through the body or the location of discomfort, although large changes can be expected with some other shocks. With the motions investigated, higher frequency motions caused discomfort in the lower body whereas lower frequency components caused discomfort in the upper body.

\subsection{Frequency weighting}

\section{Gain of the frequency weighting}

Unlike Zhou and Griffin (2014b, 2017b) the present study also investigated discomfort caused by frequencies less than $1 \mathrm{~Hz}$ (i.e., $0.5,0.63$, and $0.8 \mathrm{~Hz}$ ). When the contours for a subjective magnitude of 100 were expressed in terms of unweighted VDV, there were no significant differences between the equivalent comfort contours for vibration and shock at these lower frequencies. However, for shocks having fundamental frequencies in this range, the standardised $0.4-\mathrm{Hz}$ high-pass filter used with frequency weighting $W_{\mathrm{b}}$ attenuates some of the low frequencies present in the shock waveform and reduces the frequency-weighted VDV of the shocks. For example, if the fundamental frequency is 0.5 $\mathrm{Hz}$, a sinusoidal vibration and a 11/2-cycle shock with unweighted VDVs of $2.0 \mathrm{~ms}^{-1.75}$ have VDVs of 1.7 and $1.5 \mathrm{~ms}^{-1.75}$, respectively, after being filtered solely by the $0.4-\mathrm{Hz}$ highpass filter required when applying the $W_{\mathrm{b}}$ frequency weighting. After being frequencyweighted using $W_{\mathrm{b}}$ (with the $0.4-\mathrm{Hz}$ high-pas filter in accord with the standards), the VDVs of these two motions are 0.7 and $0.6 \mathrm{~ms}^{-1.75}$, respectively. This effect of the high-pass filter on the frequency-weighted magnitude of the shocks may explain why, at frequencies less than about $0.8 \mathrm{~Hz}$, the shocks appear to cause more discomfort than vibration with the same frequency-weighted VDV (Figure 6). The difference in frequency-weighted VDVs for vibration and shock is somewhat reduced because the 5-s sinusoidal waveforms used in this study also contained some energy at frequencies less $0.4 \mathrm{~Hz}$, and so were also slightly attenuated by the $0.4-\mathrm{Hz}$ high-pass filter.

The approach to predicting the discomfort caused by oscillatory motion has been to define a frequency weighting (from studies with differing frequencies of more-or-less sinusoidal 
oscillation of constant duration) and a duration weighting (from studies with oscillations of differing duration). The simple combination of the frequency weighting and the duration weighting (e.g., the frequency-weighted vibration dose value) assumes that the frequency weighting is independent of the duration of the motion, and that the duration weighting is independent of the frequency of the motion, and that both weightings are independent of the magnitude of the motion. Such assumptions seem necessary in order to define a simple practical method for evaluating the severity of oscillatory motion. However, the optimum frequency weighting depends on the magnitude of vibration at the moderate magnitudes of motion used in this study and at much lower magnitudes (e.g., Morioka and Griffin, 2006a). The appropriate weighting may also be expected to change at greater magnitudes than investigated in this study if contact between the seat and the body is lost at times during a cycle of motion.

The use of a simple frequency weighting (e.g., $W_{b}$ ) with a simple duration weighting (e.g., VDV) provides a practical method of evaluating shock severity, but the 'non-linearities' in human responses mean that no such weightings (or other linear and practical methods) can provide highly accurate predictions of the discomfort caused by a wide range of shocks.

\section{Phase of the frequency weighting}

When a shock is weighted (by the appropriate frequency weighting and band-pass filters defined in a standard, such as BS 6841:1987 or ISO 2631-1:1997) the waveform of the shock is distorted by the phase responses of all the filters delaying different frequencies by differing amounts. Because the phase response of filters can affect the measured value, it is important that standardised evaluations of vibration are made using filters that have the standardised phase response as well as the standardised gain.

The problem is not confined to defining the appropriate phase for the filter representing the human response (e.g. frequency weighting $W_{\mathrm{b}}$ ), it also applies to the band-limiting filters $\left(0.4-\mathrm{Hz}\right.$ high-pass filter and $100-\mathrm{Hz}$ low-pass filter used with $\left.W_{\mathrm{b}}\right)$. For shocks with the lowest and highest fundamental frequencies used in this study (i.e., 0.5 and $16 \mathrm{~Hz}$ ), the unweighted waveforms, the waveforms filtered solely by the $0.4-\mathrm{Hz}$ high-pass filter, and the weighted waveforms (weighted by frequency-weighting $W_{\mathrm{b}}$ with the designated band-pass filters at 0.4 and $100 \mathrm{~Hz}$ ) are compared in Figure 10. The shock with the 0.5 $\mathrm{Hz}$ fundamental frequency is much distorted by the high-pass filter. The phase responses of both the frequency weighting and the high-pass filter also affect the VDV of the waveform and its peak values. The $0.4-\mathrm{Hz}$ high pass filter attenuates $0.5-\mathrm{Hz}$ motion (to $84 \%$ of its value) as well as distorting the waveform. The same phenomenon will happen with motions having frequencies close to the low-pass filter at $100 \mathrm{~Hz}$. The effects of the 
band-limiting filters merit further consideration when evaluating a shock, even if the fundamental frequency of the shock is within the range 0.5 to $80 \mathrm{~Hz}$.

\section{FIGURE 10 ABOUT HERE}

\section{Conclusions}

An appropriately frequency-weighted vibration dose value seems preferable to either the peak acceleration or the r.m.s. acceleration when predicting the discomfort caused by both sinusoidal vibration and shock. The peak value does not reflect the duration of the motion or even how many times the peak value is reached and is not a reasonable general purpose measure for predicting the discomfort caused by shocks. The r.m.s. acceleration is not appropriate for evaluating shocks because the duration of measurement cannot be defined in a practically useful way.

The rate of growth of discomfort with increasing magnitude of vibration or mechanical shock depends on the fundamental frequency of the motion and differs between a sinusoidal vibration and a shock of the same fundamental frequency. The frequencydependence of discomfort caused by vibration and shock therefore varies according to the magnitude of the motion and differs between sinusoidal vibration and shock. It follows that no single frequency weighting is optimum for all magnitudes or to both types of motion.

At frequencies causing greatest discomfort, if a vibration and a shock have the same fundamental frequency and the same magnitude, the shock will cause less discomfort. Irrespective of whether the magnitudes of the two motions are expressed in terms of peak acceleration or unweighted vibration dose value, vertical shocks with fundamental frequencies in the range 4 to $16 \mathrm{~Hz}$ therefore cause less discomfort than vertical sinusoidal motions of the same frequency and the same magnitude. When their magnitudes are expressed in terms of frequency-weighted vibration dose values, the differences between the motions are reduced, but the effects of motion magnitude on the frequencydependence of discomfort remain.

With fundamental frequencies less than about $1 \mathrm{~Hz}$, shocks cause slightly greater discomfort than vibration with the same frequency-weighted VDV, because the high-pass filters employed in the frequency weighting attenuate some low frequency components in the shock waveform more than they attenuate sinusoidal vibration of the same fundamental frequency. The effects of the gain and the phase of the high-pass filter need consideration if the standardised evaluation method is used to evaluate mechanical shocks containing low frequencies. 


\section{Acknowledgements}

This work was undertaken on contract ROD - HQ SG 34/02/08/05 in association with the Institute of Naval Medicine. The support of Dr GS Paddan is gratefully acknowledged.

\section{References}

Ahn SJ and Griffin MJ (2008) Effects of frequency, magnitude, damping, and direction on the discomfort of vertical whole-body mechanical shocks. Journal of Sound and Vibration, $311,485-497$.

Basri B and Griffin MJ (2013) Predicting discomfort from whole-body vertical vibration when sitting with an inclined backrest. Applied Ergonomics, 44, 423-434.

British Standards Institution (1987) Guide to Measurement and evaluation of human exposure to whole-body mechanical vibration and repeated shock, BS 6841-1:1987.

Corbridge C and Griffin MJ (1986) Vibration and comfort: vertical and lateral motion in the range 0.5 to $5.0 \mathrm{~Hz}$. Ergonomics, 29(2), 249-272.

Dupuis H, Hartung E and Louda L (1972) The effect of random vibrations of a limited frequency band compared with sinusoidal vibrations, on human beings. Royal Aircraft Establishment, Library Translation No 1603. Ministry of Defence Farnborough, Hants.

Griffin MJ (1976) Subjective Equivalence of sinusoidal and random whole-body vibration. Journal of Acoustical Society of America 60(5), 1140-1145.

Griffin MJ, Whitham EM and Parson KC (1982) Vibration and comfort I. Translational seat vibration. Ergonomics, 25(7), 603-630.

International Organization for Standardization (1997). Mechanical vibration and shock Evaluation of human exposure to whole-body vibration - Part 1. BS ISO 2631:1997.

Jones A J and Saunders D J (1972) Equal comfort contours for whole body vertical, pulsed sinusoidal vibration, Journal of Sound and Vibration, 23,1-14.

Matsumoto Y and Griffin MJ (2002) Effect of phase on human responses to vertical wholebody vibration and shock - experimental investigation. The Journal of the Acoustical Society of America, 111(3), 1280-8.

Morioka M and Griffin MJ (2006a) Magnitude-dependence of equivalent comfort contours for fore-and-aft, lateral and vertical whole-body vibration. Journal of Sound and Vibration, $298,755-772$. 
Morioka M and Griffin MJ (2006b) Magnitude-dependence of equivalent comfort contours for fore-and-aft, lateral and vertical hand-transmitted vibration. Journal of Sound and Vibration, 295, 633-648.

Patelli G (2016) The frequency-dependence of discomfort caused by vibration and mechanical shocks - Chapter 4. In: Effects of vertical mechanical shocks and body posture on discomfort. Dissertation thesis, University of Southampton, United Kingdom.

Wyllie IH and Griffin MJ (2009) Discomfort from sinusoidal oscillation in the pitch and foreand-aft axes at frequencies between 0.2 and $1.6 \mathrm{~Hz}$. Journal of Sound and Vibration, $324(1-2), 453-467$.

Zhou Z and Griffin MJ (2014a) Response of the seated human body to whole-body vertical vibration: biodynamic responses to sinusoidal and random vibration. Ergonomics, 57(5), $693-713$.

Zhou Z and Griffin MJ (2014b) Response of the seated human body to whole-body vertical vibration: discomfort caused by sinusoidal vibration. Ergonomics, 57(5), 714-732.

Zhou Z. and Griffin M.J. (2017a) Response of the seated human body to whole-body vertical vibration: biodynamic responses to mechanical shocks. Ergonomics, 60, 3, 333346.

Zhou Z. and Griffin M.J. (2017b) Response of the seated human body to whole-body vertical vibration: discomfort caused by mechanical shocks. Ergonomics, 60, 3, 347-357. 
Table 1 Unweighted r.m.s. accelerations and peak accelerations $\left(\mathrm{ms}^{-2}\right)$ of sinusoidal vibration and 11/2-cycle shocks used in the experiment (Patelli, 2016).

\begin{tabular}{|c|c|c|c|c|c|c|c|c|c|c|c|c|c|c|c|c|}
\hline \multirow{2}{*}{\begin{tabular}{|l}
$\begin{array}{c}\text { Frequency } \\
(\mathrm{Hz})\end{array}$ \\
0.5 \\
\end{tabular}} & \multicolumn{8}{|c|}{$\begin{array}{c}\text { Sinusoidal vibration } \\
\text { unweighted r.m.s. acceleration } \\
\left(\mathrm{ms}^{-2}\right)\end{array}$} & \multicolumn{8}{|c|}{$\begin{array}{c}11 / 2 \text {-cycle shocks } \\
\text { unweighted peak acceleration } \\
\left(\mathrm{ms}^{-2}\right)\end{array}$} \\
\hline & 0.3 & 0.3 & 0.4 & 0.5 & 0.7 & 0.9 & 1.1 & 1.4 & 0.7 & 0.8 & 1.0 & 1.3 & 1.6 & 2.0 & 2.6 & 3.2 \\
\hline 0.63 & 0.3 & 0.7 & 0.5 & 0.6 & 0.0 & 1.0 & 1.2 & 1.5 & 0.8 & 1.0 & 1.2 & 1.6 & 2.0 & 2.4 & 3.1 & 3.9 \\
\hline 0.8 & 0.5 & 0.6 & 0.7 & 0.9 & 1.2 & 1.4 & 1.8 & 2.3 & 1.2 & 1.6 & 2.0 & 2.5 & 3.1 & 3.9 & 4.9 & 6.2 \\
\hline 1.0 & 0.6 & 0.7 & 0.9 & 1.1 & 1.4 & 1.8 & 2.3 & 2.8 & 1.6 & 2.0 & 2.5 & 3.2 & 4.0 & 5.0 & 6.4 & 8.0 \\
\hline 1.25 & 0.6 & 0.7 & 0.9 & 1.1 & 1.4 & 1.8 & 2.3 & 2.8 & 1.7 & 2.1 & 2.6 & 3.3 & 4.1 & 5.2 & 6.6 & 8.2 \\
\hline 1.6 & 0.6 & 0.7 & 0.9 & 1.1 & 4 & 1.7 & 2.2 & 2.8 & 1.7 & 2.1 & 2.6 & 3.3 & 4.1 & 5.2 & 6.6 & 8.2 \\
\hline 2.0 & 0.7 & 0.8 & 1.0 & 1.3 & 1.6 & 2.1 & 2.6 & 3.3 & 1.9 & 2.4 & 3.1 & 3.9 & 4.8 & 6.2 & 7.7 & 8.7 \\
\hline 2.5 & 0.6 & 0.7 & 0.9 & 1.1 & 1.4 & 1.8 & 2.2 & 2.8 & 1.7 & 2.1 & 2.7 & 3.4 & 4.2 & 5.4 & 6.8 & 8.4 \\
\hline 3.15 & 0.5 & 0.7 & 0.8 & 1.0 & 1.3 & 1.7 & 2.1 & 2.6 & 1.8 & 2.3 & 2.9 & 3.6 & 4.6 & 5.7 & 7.2 & 9.0 \\
\hline 4.0 & 0.4 & 0.5 & 0.6 & 0.8 & 1.0 & 1.2 & 1.5 & 1.9 & 1.6 & 2.0 & 2.5 & 3.2 & 4.1 & 5.1 & 6.3 & 7.9 \\
\hline 5.0 & 0.4 & 0.5 & 0.7 & 0.9 & 1.1 & 1.3 & 1.7 & 2.1 & 1.9 & 2.4 & 3.0 & 3.9 & 4.8 & 6.0 & 7.5 & 9.4 \\
\hline 6.3 & 0.4 & 0.5 & 0.7 & 0.8 & 1.0 & 1.3 & 1.6 & 2.0 & 1.9 & 2.4 & 3.0 & 3.9 & 4.8 & 6.1 & 7.6 & 9.5 \\
\hline 8.0 & 0.3 & 0.4 & 0.5 & 0.7 & 0.9 & 1.1 & 1.3 & 1.7 & 1.6 & 2.1 & 2.6 & 3.2 & 4.1 & 5.1 & 6.4 & 8.0 \\
\hline 10 & 0.4 & 0.5 & 0.6 & 0.7 & 0.9 & 1.1 & 1.4 & 1.8 & 1.8 & 2.2 & 2.8 & 3.5 & 4.5 & 5.6 & 7.0 & 8.7 \\
\hline 12.5 & 0.3 & 0.4 & 0.5 & 0.6 & 0.8 & 1.0 & 1.2 & 1.5 & 1.6 & 2.0 & 2.5 & 3.1 & 3.9 & 5.0 & 6.3 & 7.8 \\
\hline 16 & 0.3 & 0.3 & 0.4 & 0.5 & 0.7 & 0.9 & 1.1 & 1.4 & 1.5 & 1.8 & 2.3 & 2.9 & 3.7 & 4.6 & 5.9 & 7.3 \\
\hline
\end{tabular}


Table 2 The rate of growth, $n$, and the constant, $k$, obtained in this study (median values for 17 subjects). For both the sinusoidal vibration and the shocks the value of $k$ applies to unweighted vibration dose values. The value of $n$ is independent of the measure of vibration magnitude. Based on Table 4.2 in Patelli (2016).

\begin{tabular}{|c|c|c|c|c|}
\hline \multirow{2}{*}{$\begin{array}{c}\text { Frequency } \\
(\mathrm{Hz})\end{array}$} & \multicolumn{2}{|c|}{ Sinusoidal vibration } & \multicolumn{2}{c|}{$11 / 2$-cycle shock } \\
\cline { 2 - 5 } & Exponent $n$ & Constant $k$ & Exponent $n$ & Constant $k$ \\
\hline 0.5 & 1.21 & 51.29 & 1.43 & 65.00 \\
\hline 0.63 & 1.17 & 52.99 & 1.34 & 47.65 \\
\hline 0.8 & 0.82 & 60.83 & 1.18 & 39.14 \\
\hline 1 & 0.73 & 63.93 & 0.97 & 52.71 \\
\hline 1.25 & 0.73 & 54.92 & 0.79 & 48.93 \\
\hline 1.6 & 0.64 & 48.81 & 0.68 & 48.38 \\
\hline 2 & 0.69 & 53.55 & 0.82 & 57.22 \\
\hline 2.5 & 0.74 & 48.35 & 0.93 & 50.03 \\
\hline 3.15 & 0.68 & 61.26 & 0.90 & 52.50 \\
\hline 4 & 0.74 & 84.46 & 0.91 & 62.09 \\
\hline 5 & 0.48 & 102.71 & 0.77 & 62.60 \\
\hline 6.3 & 0.62 & 96.24 & 0.81 & 56.93 \\
\hline 8 & 0.56 & 92.99 & 0.71 & 67.13 \\
\hline 10 & 0.61 & 91.98 & 0.77 & 61.65 \\
\hline 12.5 & 0.47 & 101.99 & 0.64 & 49.01 \\
\hline 16 & 0.39 & 95.55 & 0.77 & 52.52 \\
\hline
\end{tabular}



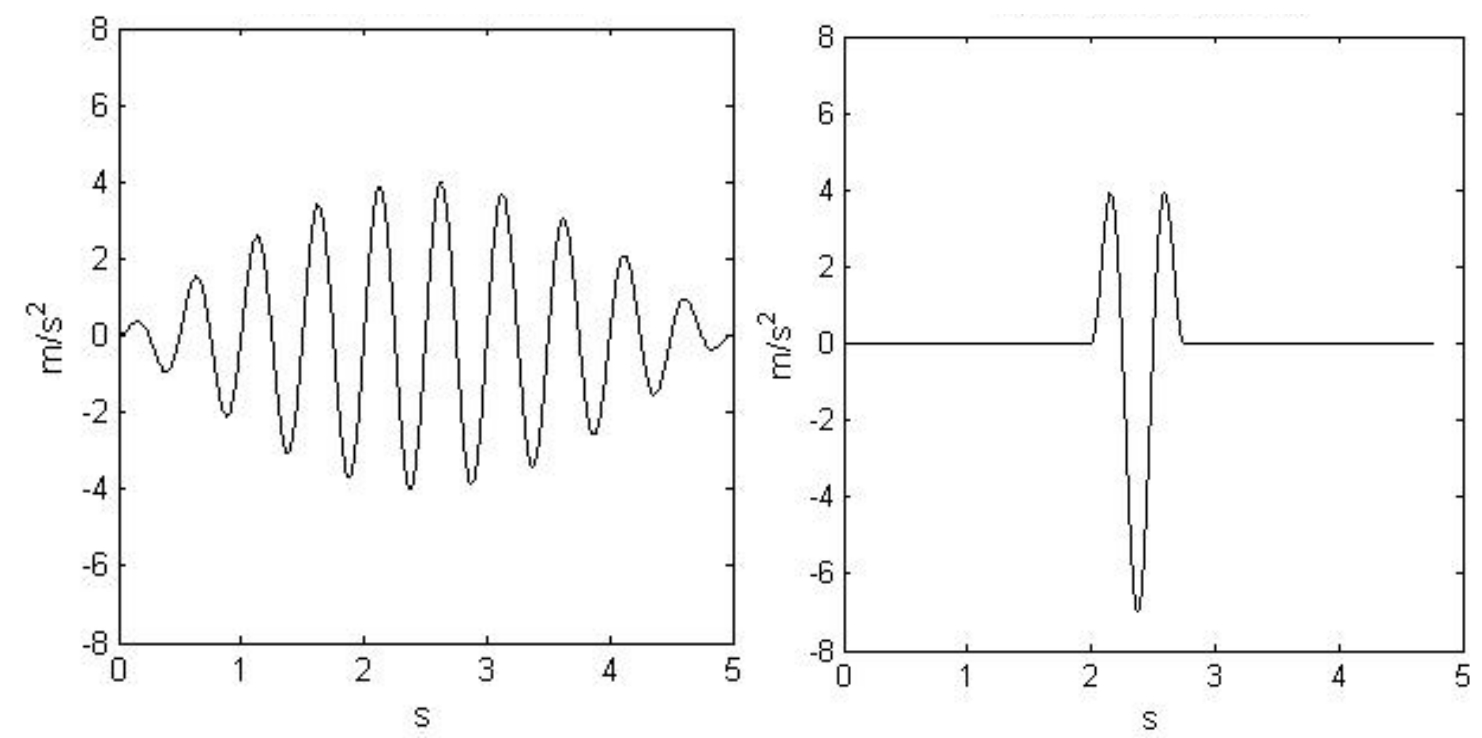

Figure 1 Enveloped 2-Hz sinusoidal acceleration waveform (left) and 11/2-cycle 2$\mathrm{Hz}$ shock acceleration waveform (right) used in the study; from Figure 4.1 in Patelli (2016). 

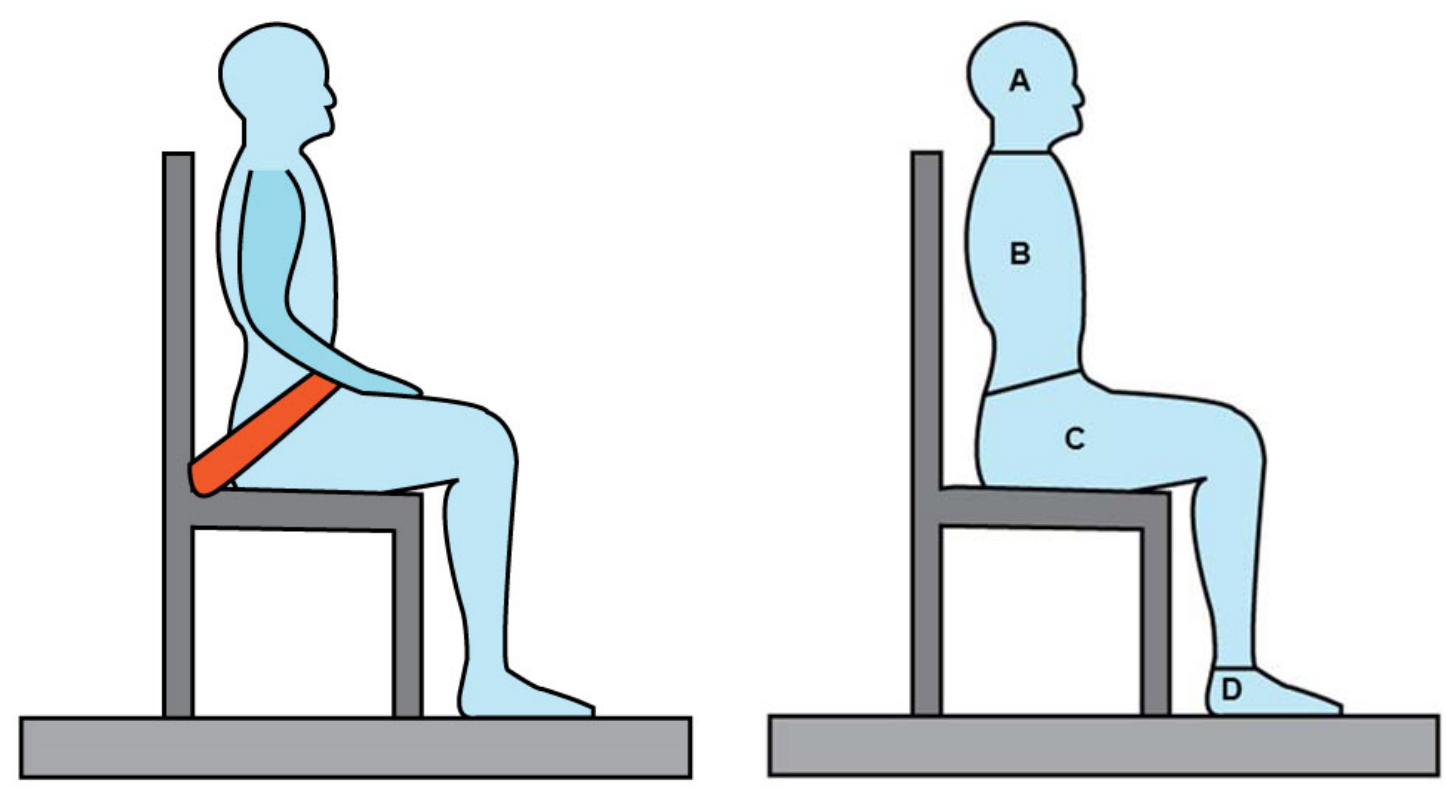

Figure 2. Posture adopted by subjects: sitting upright with a loose lap belt for safety and no contact with the backrest (left). Body map used for indicating the locations of most discomfort (right). From Figure 4.2 in Patelli (2016). 


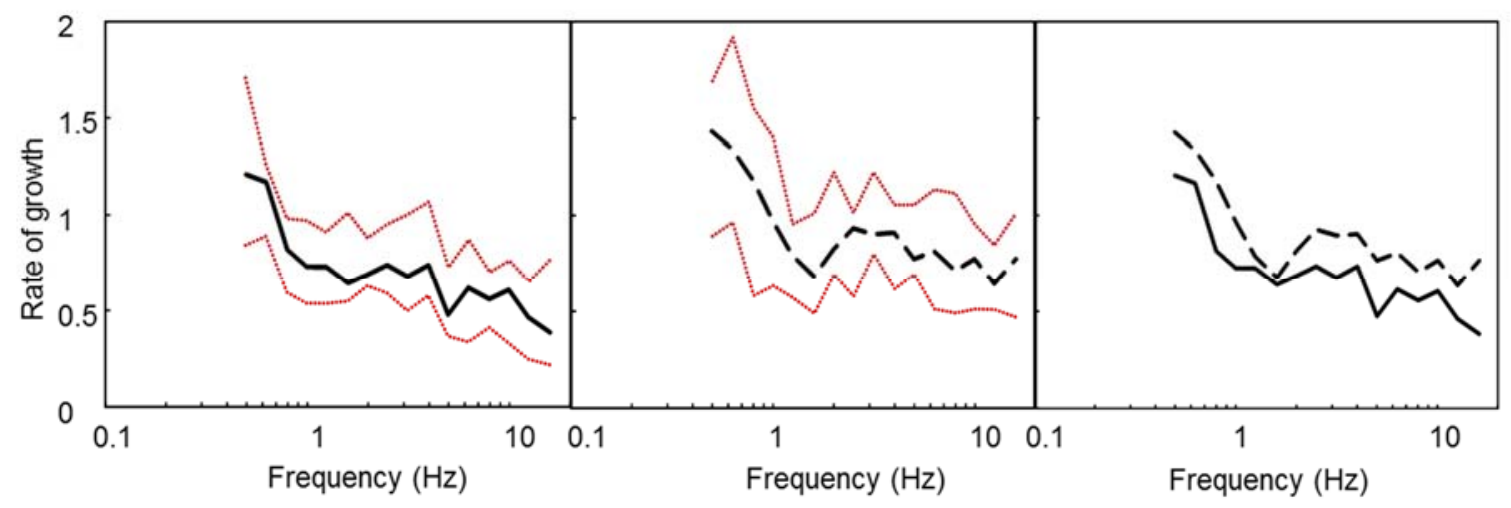

Figure 3 Rate of growth of discomfort, $n$, for vibration (-) and shocks (- - ) with inter-quartile ranges ( …......). Median values for 17 subjects. Based on Figure 4.3 in Patelli (2016). 


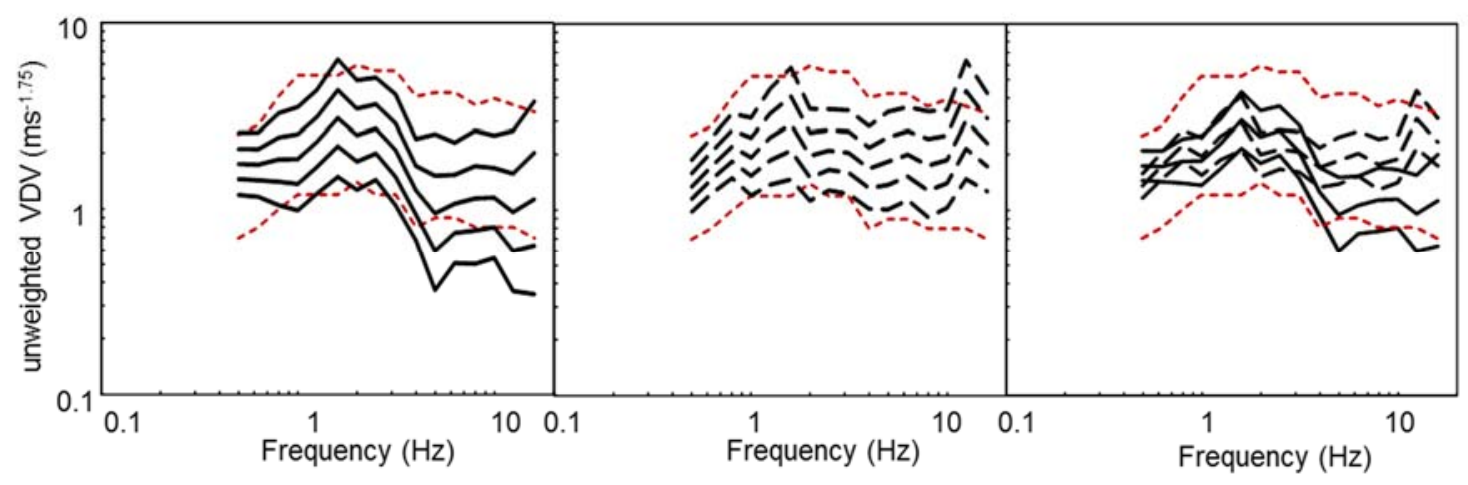

Figure 4 Equivalent comfort contours for vibration (-) and shocks (- - in terms of unweighted vibration dose values, VDV. Contours correspond to subjective magnitudes: $\psi=63,80,100,125$ and 160 (left and middle figure) and $\psi=100,125$ and 160 (right figure), where a subjective magnitude of 100 corresponds to the discomfort experienced with a 5-s sinusoidal motion with a frequency of $2.5 \mathrm{~Hz}$ and an unweighted VDV of $2.0 \mathrm{~ms}^{-1.75}$. Minimum and maximum magnitudes employed in the study (- - - -). Medians for 17 subjects. Based on Figures 4.4 and 4.5 in Patelli (2016). 


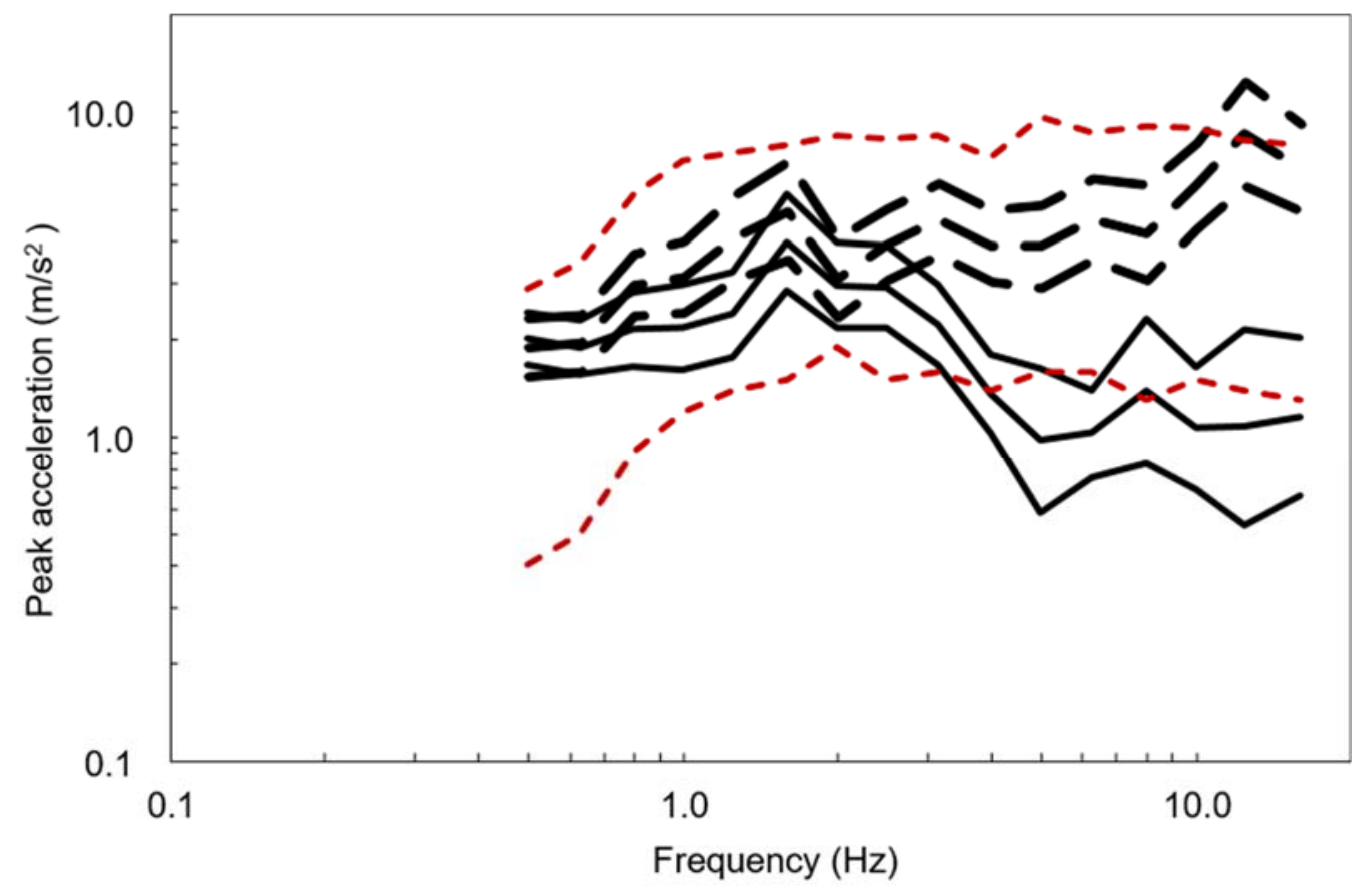

Figure 5 Equivalent comfort contours for vibration (-) and shocks ( $-(-)$ in terms of unweighted peak acceleration $\left(\mathrm{ms}^{-2}\right)$. Contours corresponding to subjective magnitudes $\psi=80,100$, and 125 are displayed, where a subjective magnitude of 100 corresponds to the discomfort experienced with a 5-s sinusoidal motion with a frequency of $2.5 \mathrm{~Hz}$ and an unweighted acceleration of $1.1 \mathrm{~ms}^{-2}$ r.m.s.. Minimum and maximum magnitudes of shock employed in the study (- - - ). Medians for 17 subjects. Based on Figure 4.6 in Patelli (2016). 


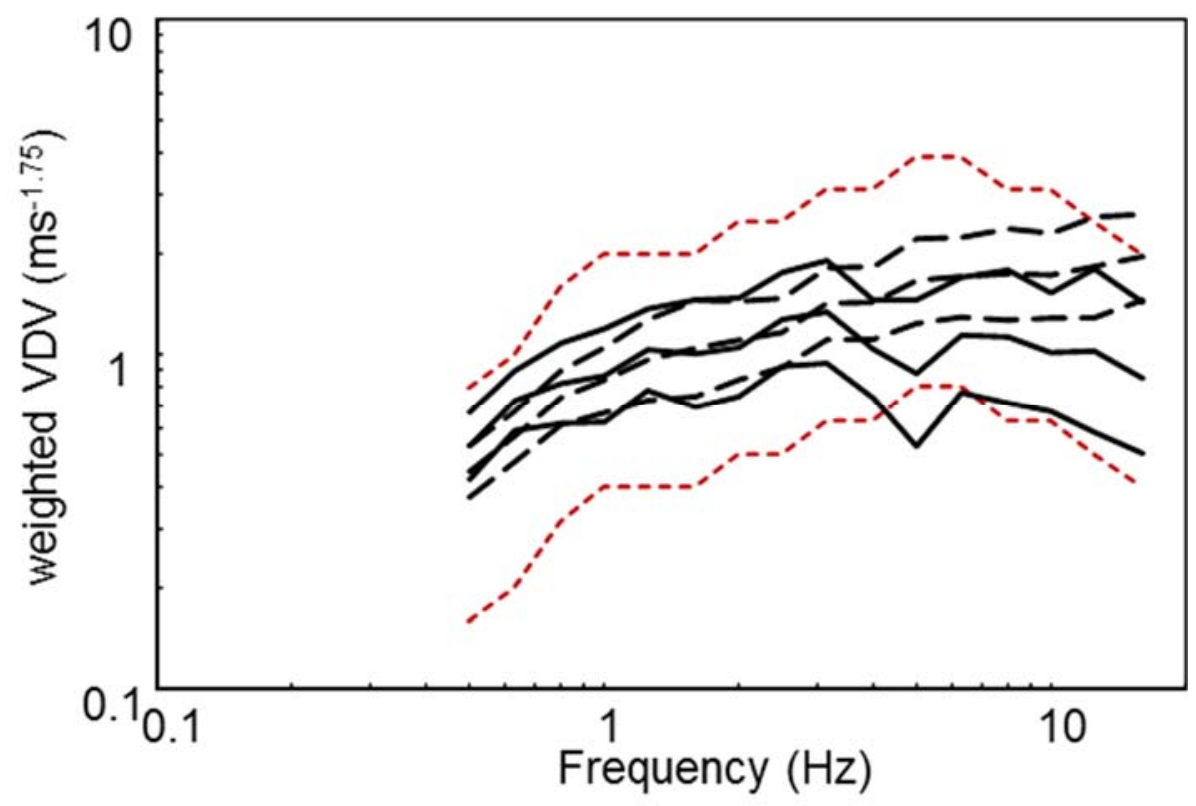

Figure 6 Comparisons of equivalent comfort contours for frequency- weighted VDV obtained with vibration (-) and shock (- -) for three subjective magnitudes: $\psi=80,100$ and 125, where a subjective magnitude of 100 corresponds to the discomfort experienced with a 5-s sinusoidal motion with a frequency of $2.5 \mathrm{~Hz}$ and $W_{\mathrm{b}}$-weighted VDV of $\left.1.0 \mathrm{~ms}^{-1.75}\right)$. Medians for 17 subjects. Based on Figure 4.7 in Patelli (2016). 
Figure 7 Dependence of the location of discomfort on the frequency and magnitude of vertical vibration and vertical shock. Lowest magnitude $\left(0.5 \mathrm{~ms}^{-2}\right.$ unweighted r.m.s. at $2.5 \mathrm{~Hz})$, middle magnitude $\left(1.1 \mathrm{~ms}^{-2}\right.$ unweighted r.m.s. at $2.5 \mathrm{~Hz})$ and at the highest magnitude of vibration $\left(2.8 \mathrm{~ms}^{-2}\right.$ unweighted r.m.s. at $2.5 \mathrm{~Hz}$ ). Body locations as in Figure 2. Based on Figure 4.8 in Patelli (2016). 


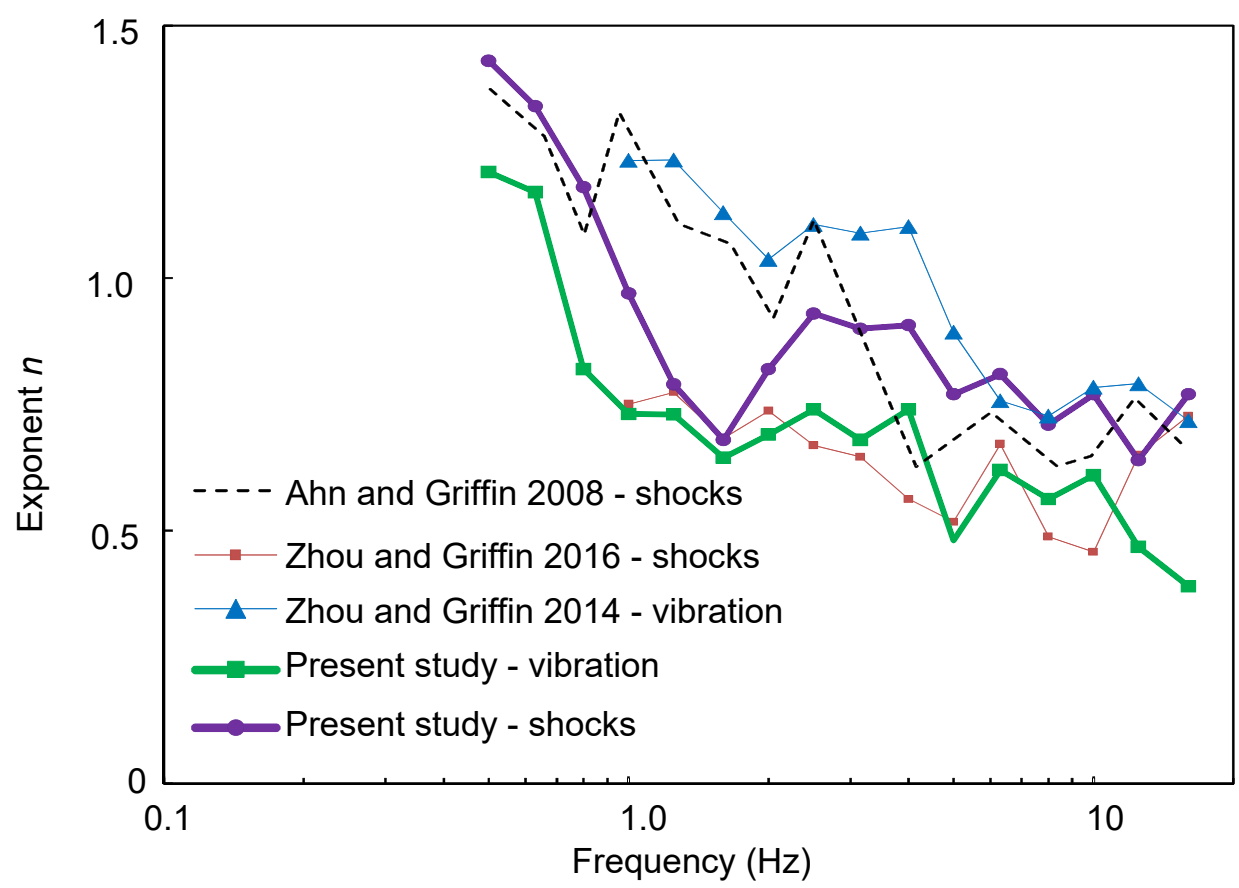

Figure 8 Comparison of the rates of growth of discomfort obtained with low frequency sinusoidal vibration and shock in the present and previous studies. Developed from Figure 4.9 in Patelli (2016). 

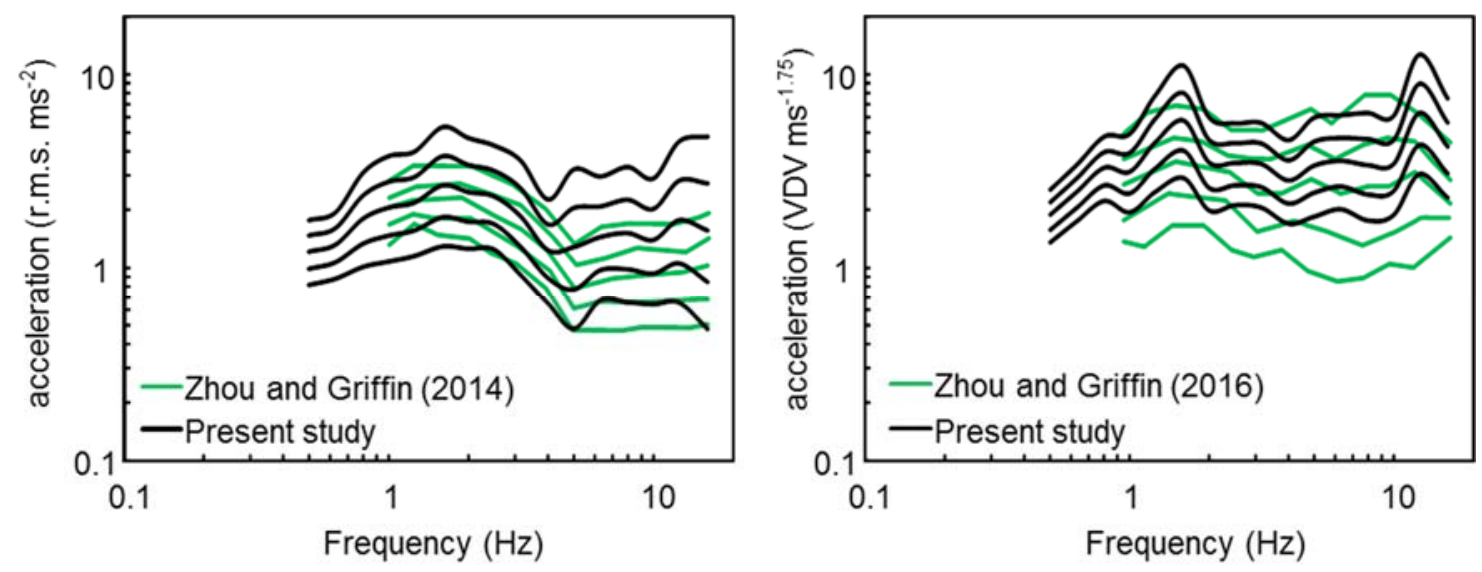

Figure 9 Comparison between equivalent comfort contours. Left: sinusoidal vibration in terms of unweighted r.m.s. acceleration from this study and Zhou and Griffin (2014); right: shock in terms of unweighted VDV from this study and Zhou and Griffin (2016). Contours are shown for subjective magnitudes of 100, 125, 160,200 , and 250 . The subjective magnitudes differ between the studies but the shapes of the contours can be compared. Based on Figure 4.11 in Patelli (2016). 


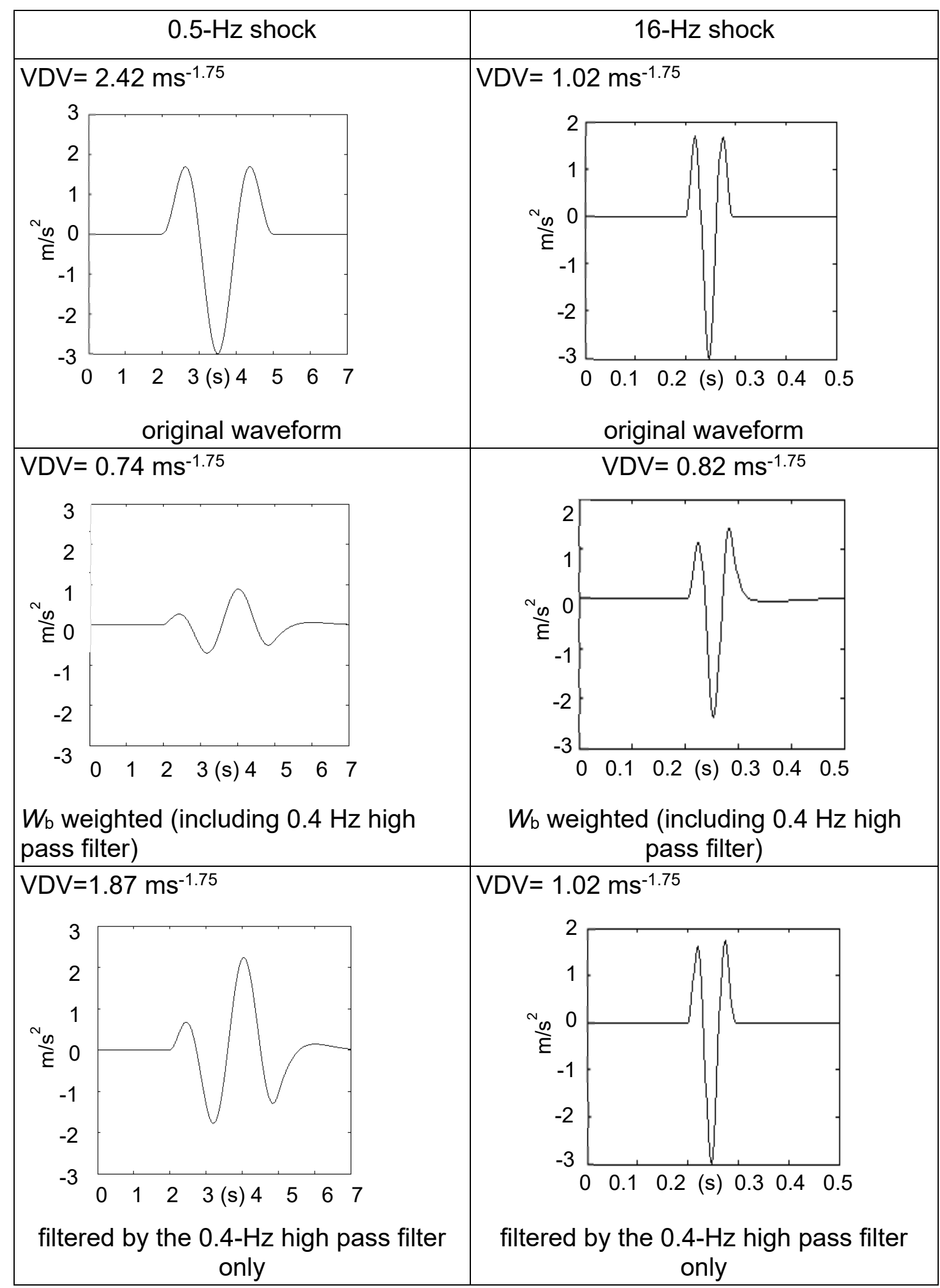

Figure 10 Effect of frequency weighting $W_{\mathrm{b}}$ and $0.4-\mathrm{Hz}$ high-pass filter on shock waveforms. Left: a shock with fundamental frequency of $0.5 \mathrm{~Hz}$. Right: a shock with fundamental frequency of $16 \mathrm{~Hz}$. Based on Figure 4.13 in Patelli (2016). 